\title{
IncRNA SNHG3 acts as a novel Tumor Suppressor and regulates Tumor Proliferation and Metastasis via AKT/mTOR/ERK pathway in Papillary Thyroid Carcinoma
}

\author{
Yu Duan¹, Zhiyong Wang², Lijuan $\mathrm{Xu}^{1}$, Li Sun ${ }^{1}$, Hairong Song1, Huiqing Yin ${ }^{1}$, Fucheng $\mathrm{He}^{1 凶}$ \\ 1. Department of Medical Laboratory, The First Affiliated Hospital of Zhengzhou University, Zhengzhou, Henan 450000, China. \\ 2. Department of Urology, The First Affiliated Hospital of Zhengzhou University, Zhengzhou, Henan 450000, China. \\ $\triangle$ Corresponding author: Fucheng He, Department of Medical Laboratory, The First Affiliated Hospital of Zhengzhou University, No. 1 East Jianshe Road, \\ Zhengzhou Henan 450000, China. E-mail: hefucheng@zzu.edu.cn.
}

() The author(s). This is an open access article distributed under the terms of the Creative Commons Attribution License (https://creativecommons.org/licenses/by/4.0/). See http://ivyspring.com/terms for full terms and conditions.

Received: 2019.11.13; Accepted: 2020.01.19; Published: 2020.03.15

\begin{abstract}
The incidence of papillary thyroid carcinoma (PTC) has been increased rapidly in recent decades. Long noncoding RNAs (IncRNA) are a class of non-protein-coding transcripts and play critical roles in regulating gene expression and influence biological behaviors of multiple cancers, including PTC. Here, we discovered that IncRNA SNHG3 was significantly downregulated in PTC tissues and cell lines, the expression of SNHG3 was negatively correlated with the TNM stage and poor prognosis of PTC patients. Functional studies illustrated that the depletion of SNHG3 via CRISPR/Cas9 technology promoted the proliferation, migration and invasion abilities of PTC cells. Tumor xenograft models confirmed the tumor-promoting role of silenced SNHG3 in vivo. Further mechanistic analyses revealed that knockout of SNHG3 activated the AKT/mTOR/ERK pathway in PTC cell lines and the mTOR inhibitor AZD8055 abrogated the tumor-promoting effect induced by SNHG3 inhibition. Taken together, our findings identified a IncRNA SNHG3 that functions its tumor-suppressor role during PTC development and SNHG3 might serve as a promising candidate for target therapy of PTC.
\end{abstract}

Key words: Papillary thyroid cancer, Long non-coding RNA, SNHG3, mTOR, AZD8055

\section{Introduction}

Thyroid cancer is the most prevalent endocrine malignancy and the fifth most common cancer in women, ranking in ninth place for the global cancer incidence, with approximately 570000 new cases annually around the world [1]. Thyroid cancer can be categorized into four groups based on pathology type, papillary thyroid carcinoma (PTC) of which is the most frequently diagnosed type, accounting for $90 \%$ cases of thyroid cancers [2]. Most patients with PTC have an overall 5-year survival rate above 90\%; however, about $15 \%$ of patients exhibited poor prognosis owing to the tumor metastasis [3]. Although the genomic landscape of PTC has been extensively characterized, the intrinsic-factors that control thyroid cancer development are less well understood. Therefore, identifying the novel targets for thyroid tumorigenesis and progression is of particular interest.

Long noncoding RNAs (lncRNAs) are transcripts with over 200 nucleotides in length without proteincoding capabilities. Although lncRNAs had been regarded as the "desert region" of the genome for many years, recently increasing evidence has disclosed that lncRNAs play critical roles in regulating gene expression at various aspects, including mRNA alternative splicing, protein activities, alteration of protein localization and epigenetic programming [4,5]. A variety of lncRNAs are aberrantly expressed in specific cancer types [6]. Notably, the underlying mechanisms of lncRNAs contributing to cancer development are diverse [7]. They are involved in human malignancies through 
driving many crucial cancer phenotypes, including cell cycle regulation, survival, immune response and pluripotency in cancer cells [8]. For instance, Kawasaki et al. identified a lncRNA, MYU, that mediated the induction of CDK6 to promote cell cycle progression in colon cancer cells [9]. IncRNA-Hh strengthens cancer stem cell generation in breast cancer via activation of the hedgehog signaling pathway [10].

Small nucleolar host gene 3 (SNHG3) is a novel lncRNA located at 1p36.1. SNHG3 was firstly implicated in Alzheimer's disease (AD) and its upregulation might be an indicator of a wider dysregulation of translational machinery and ribosome biogenesis during $\mathrm{AD}$ neurodegeneration [11]. Subsequently, it was revealed to be an oncogene and a biomarker of malignant status or poor prognosis in several types of cancers, including colorectal cancer, ovarian cancer and hepatocellular carcinoma [12-14]. However, no studies to date reported its potential tumor suppressor function in cancer.

In the current study, we revealed that SNHG3 was silenced in PTC tissues and cell lines and inhibition of SNHG3 via CRISPR/Cas9 facilitated PTC cell proliferation, migration and invasion. Mechanistic studies demonstrated that SNHG3 promoted PTC malignant progression through AKT/ mTOR/ERK signaling pathway. Furthermore, blockade of mTOR by AZD8055 hampered the tumorpromoting effect from the loss of SNHG3. To the best of knowledge, our study, for the first time, highlighted the pivotal role of SNHG3 as a tumor suppressor in PTC tumorigenicity.

\section{Materials and Methods}

\section{Human PTC samples collection}

All samples were obtained from the First Affiliated Hospital of Zhengzhou University between January 2017 and December 2018, who diagnosed with PTC. Histopathologic diagnoses were confirmed by the pathologists and classified based on the 7th American Joint Committee on Cancer (AJCC) staging system. Patients were excluded from study if their thyroid malignancy was not of papillary histology or if they received treatment with radioiodine therapy or chemotherapy before surgery. All patients were provided with written informed consent and were treated with total or near-total thyroidectomy for PTC in surgery. This study procedure was approved by the medical ethics committee of the First Affiliated Hospital of Zhengzhou University.

\section{Cell culture}

Human immortalized thyroid cells Nthy-ori 3-1 and human PTC cell lines BCPAP, KTC- 1 and TPC-1 were purchased from the Stem Cell Bank, Chinese Academy of Science (Shanghai, China). All cell lines were maintained in high glucose DMEM (BI, Israel) supplemented with $10 \%$ FBS, 100 units $/ \mathrm{mL}$ of penicillin and $100 \mu \mathrm{g} / \mathrm{ml}$ of streptomycin at $37^{\circ} \mathrm{C}$ in $5 \% \mathrm{CO}_{2}$ atmosphere. All cells were confirmed without mycoplasma infection.

\section{Cytoplasmic and nuclear fractionation}

The nuclear and cytoplasmic fractions of cells were isolated by the invent Minute ${ }^{\mathrm{TM}}$ Cytoplasmic nuclear separation kit (Invent Biotechnologies) following the protocols of the manufacturer and treated with TRIzol reagent to obtain RNA. RNA levels of SNHG3, U1 and $\beta$-actin in the nuclear and cytoplasmic fractions were detected by qPCR. $\beta$-actin was as a cytoplasmic control, and U1 was as a nuclear control.

\section{Real-time PCR}

Total RNAs were isolated from tissues or cells using TRIzol reagent (Invitrogen Carlsbad, CA, USA). For quantification, cDNAs were synthesized by Prime Script RT Reagent Kit (Takara, Tokyo, Japan), followed by real-time quantitative PCR using SYBR Premix Ex Taq ${ }^{\mathrm{TM}}$ (Takara, Tokyo, Japan). Primer sequences were listed as follows: SNHG3 (Forward, 5'-GGAAATAAAGCTGGGCCTCG-3' and reverse, 5'AACAGAGCGACTCCATCTCC-3'), U1 (Forward, 5'GACGGGAAAAGATTGAGCGG $-3^{\prime}$ and reverse, 5'-GCCACGAAGAGAGTCTTGAAGG-3'), $\quad \beta$-actin (Forward, 5'- CATGTACGTTGCTATCCAGGC -3' and reverse, 5'- CTCCTTAATGTCACGCACGAT -3'). Gene expression was normalized to the housekeeping gene $\beta$-actin and analyzed according to the relative quantification method $\left(2^{-\Delta \Delta C t)}\right.$.

\section{CRISPR/Cas9-mediated knockout of SNHG3}

For stable knock-out of SNHG3 in cells, a pair of guide RNAs targeting SNHG3 were cloned into lentiCRISPR v2 vectors, sgRNA sequences targeting SNHG3 were listed as follows: (Forward, 5'-CACCGG ACGGGCCTGGGCCAGAAG-3' and reverse, 5'-AAA CCTTCTGGCCCAGGCCCGTCC-3'). HEK293T cells were transfected with the plasmids using Lipofectamine 2000 (Invitrogen, Carlsbad, CA, USA) reagents. 48 hours after transfection, viral supernatant was collected, filtered through a $0.45-\mu \mathrm{m}$ filter, and then added to BCPAP and TPC-1 cells. Stable cell lines were selected using puromycin $(2.0 \mu \mathrm{g} / \mathrm{mL})$ for two weeks and the knock-out efficiency was validated by RT-PCR. 


\section{Western blot}

Cells were collected and lysed in RIPA (Yeasen, Shanghai, China) with protease inhibitor cocktail (Sigma-Aldrich, St.Louis, MO, USA). Equivalent protein was loaded onto 10\% SDS-PAGE gel and transferred into nitrocellulose membranes (PALL, NewYork, USA). The blots were incubated with specific primary antibodies, followed by secondary antibodies. The proteins were visualized with the Clinx ChemiScope (Clinx Science Instruments, Shanghai, China). The antibodies used for western blot were listed as follows: anti-AKT antibodies (\#9272, Cell Signaling Technology), anti-p-AKT antibodies (\#4060, Cell Signaling Technology), antimTOR antibodies (\#2983, Cell Signaling Technology), anti-p-mTOR antibodies (\#5536, Cell Signaling Technology), anti-ERK antibodies (\#4695, Cell Signaling Technology), anti-p-ERK antibodies (\#4370, Cell Signaling Technology), anti-GAPDH antibodies (\#5174, Cell Signaling Technology).

\section{Cell viability assays}

For CCK-8 assay, $2 \times 10^{3}$ Cells were seeded on the 96-well plate, and 4 replicate wells were set. $10 \mu \mathrm{l}$ of CCK8 was added into the 96-well plate. After incubating at $37^{\circ} \mathrm{C}$ for 2 hours, absorbance at the wavelength of $450 \mathrm{~nm}$ was detected for the growth curve. For EdU assay, $5 \times 10^{3}$ cells were seeded on the 96-well plate and assessed using an EdU Labeling Kit (RiboBio, China) according to the manufacturer's instructions. Briefly, EdU labeling media were added to the plates and they were incubated for 2 hours at $37^{\circ} \mathrm{C}$. After treatment with $4 \%$ paraformaldehyde and $0.5 \%$ Triton X-100, cells were stained with anti-EdU working solution. DAPI was used to label cell nuclei. For colony formation assay, parental or SNHG3knockout cells (500 cells/well) were plated into the six-well plate and cultured for two weeks. Cells on the plates were fixed with $4 \%$ paraformaldehyde and stained with crystal violet.

\section{Transwell migration and invasion assay}

The 24-well transwell chambers (Corning, Carlsbad, CA, USA) were used to measure cell migration (without Matrigel) and invasion (with Matrigel). $1 \times 10^{5}$ cells were resuspended in $200 \mu \mathrm{l}$ serum-free medium and seeded onto the upper chambers. Mitomycin C (Sigma) was used to inhibit cell proliferation. The lower wells were filled with $700 \mu 11640$ or DMEM medium with $20 \%$ fetal bovine serum. Incubating at $37^{\circ} \mathrm{C}$ for $24 \mathrm{~h}$ (migration) or $48 \mathrm{~h}$ (invasion), the migrated or invaded cells were fixed in $4 \%$ paraformaldehyde for $20 \mathrm{~min}$ and stained with $0.1 \%$ crystal violet for $20 \mathrm{~min}$.

\section{Xenograft mouse model}

Five-week-old female BALB/c nude mice were purchased from the Vital River Laboratory Animal Technology and housed under pathogen-free conditions at $22^{\circ} \mathrm{C}$ in the $12 \mathrm{~h}$ light/ dark cycle. Animal protocols were performed according to the guidelines of Animal Ethics Committee of the First Affiliated Hospital of Zhengzhou University. Briefly, ten mice were randomly divided into scramble and SNHG3$\mathrm{KO}$ group and subcutaneously injected with $5 \times 10^{6}$ BCPAP cells/mouse in $150 \mu \mathrm{l}$ PBS into their right flanks. Tumor volumes were monitored every week with digital caliper using the following formula: $\left(\right.$ length $\times$ width $\left.^{2}\right) / 2$. Four weeks after injection, the mice were sacrificed and tumors were extracted for further studies.

\section{Statistical analysis}

All statistical analyses were performed by paired two-tailed Student's t-test or Chi-Square test with GraphPad 7.0 Software (La Jolla, CA, USA). Results were displayed as mean \pm SD from three independent experiments. P-value $<0.05$ was considered to be statistically significant.

\section{Results}

\section{SNHG3 is frequently downregulated in PTC tissues}

To address the role of SNHG3 in PTC carcinogenesis, we firstly evaluated the SNHG3 expression in the GEPIA platform (http://gepia. cancer-pku.cn/index.html) and the GEO datasets, the bioinformatics analysis results suggested that SNHG3 showed relatively low expression in PTC tissues versus normal thyroid tissues (Fig. 1A and Supplementary Fig. 1A). Next, we performed RT-PCR to examine the SNHG3 level in a cohort of 62 paired human PTC specimens and their matched normal thyroid tissues to validate the data-mining findings. We found that $38 / 62(61.3 \%)$ of the PTC patients exhibited more than a 1.5-fold decrease of SNHG3 expression in PTC tissues than matched normal thyroid tissues (Table 1 and Fig. 1B). Notably, the deficient level of SNHG3 expression was closely related to the advanced TNM stage of PTC patients (Fig. 1C). Consistently, SNHG3 expression was also lower in three PTC cell lines than normal thyroid follicular cells (Fig. 1D). Moreover, by analyzing the Kaplan-Meier Plotter dataset, low SNHG3 expression was found to be significantly associated with poor clinical outcomes, especially the recurrencefree survival (RFS) in PTC patients (Fig. 1E). Taken together, our results indicated that the decreased 
expression of SNHG3 might act as a tumor suppressor role and predict poor prognosis in PTC patients.

Table 1. Correlation between mRNA expression of SNHG3 and clinical characteristics in 62 PTC patients

\begin{tabular}{llllll}
\hline Variables & $\begin{array}{l}\text { No. of } \\
\text { patients }\end{array}$ & \multicolumn{2}{l}{ SNHG3 expression } & Chi-Square $P$-value \\
\cline { 4 - 6 } & & High group & Low group & & \\
\hline Gender & 23 & 9 & 14 & 0.0027 & 0.9583 \\
Male & 39 & 15 & 24 & & \\
Female & & & & & \\
Age & 29 & 11 & 18 & 0.0139 & 0.9061 \\
$<45$ & 33 & 13 & 20 & & \\
$\geq 45$ & & & & & \\
T stage & 36 & 16 & 20 & 1.190 & 0.2753 \\
T1 & 26 & 8 & 18 & & \\
T2+T3+T4 & & & & & \\
N stage & 28 & 9 & 19 & 0.9280 & 0.3354 \\
N0 & 34 & 15 & 19 & & \\
N1a+N1b & & & & & \\
TNM stage & 38 & 19 & 19 & 5.274 & $0.0216^{*}$ \\
I+II & 24 & 5 & 19 & & \\
III+IV & & & & & \\
Multifocality & & 11 & 13 & 0.8376 & 0.3601 \\
Yes & 24 & 13 & 25 & & \\
No & 38 & & & &
\end{tabular}

\section{Loss of SNHG3 promotes the proliferation and migration abilities of PTC cells}

The strong association of SHNG3 expression with the advanced tumor stage and poor prognosis of PTC patients prompted us to investigate whether
SNHG3 was required for PTC cell growth and metastasis. Therefore, we generated SNHG3knockout cell lines by CRISPR/Cas9 technology in BCPAP (low level of SHNG3) and TPC-1 (high level of SHNG3) background to inhibit the endogenous RNA level of SNHG3. The silencing of SNHG3 was confirmed by qRT-PCR (Fig. 2A). Moreover, through a cytoplasmic and nuclear RNA isolation assay, we found that over $90 \%$ of SNHG3 distributed in the nucleus of both BCPAP and TPC1 cells (Supplementary Fig. 2A). We further analyzed the impact of SNHG3 on the growth of PTC cells by CCK8, EdU incorporation experiment and colony formation assays. SNHG3 deficiency obviously accelerated the cell proliferation of PTC cells, as judged by CCK-8 growth curves (Fig. 2B). As shown in Fig. 2C, knock-out of SNHG3 displayed larger and more colonies compared with the control group. Besides, the results from the EdU test indicated that inhibition of SNHG3 enhanced the viability of PTC cells (Fig. 2D). These results supported the notion that SNHG3 regulated PTC cell proliferation in vitro. Next, we performed matrigel-coated and uncoated transwell assays to determine the effect of SNHG3 on the metastasis of PTC cells. The transwell results showed that depletion of SNHG3 remarkably elevated the invasive and migratory capabilities of PTC cells, as evidenced by an increased number of

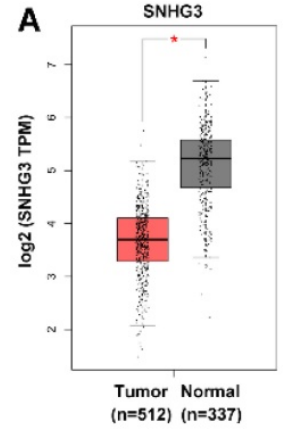

D

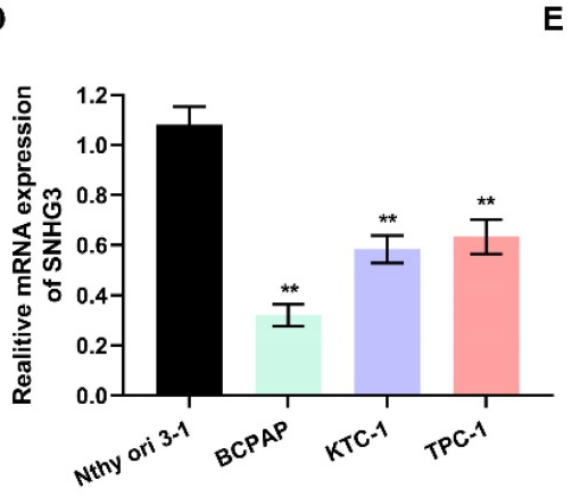

B

E
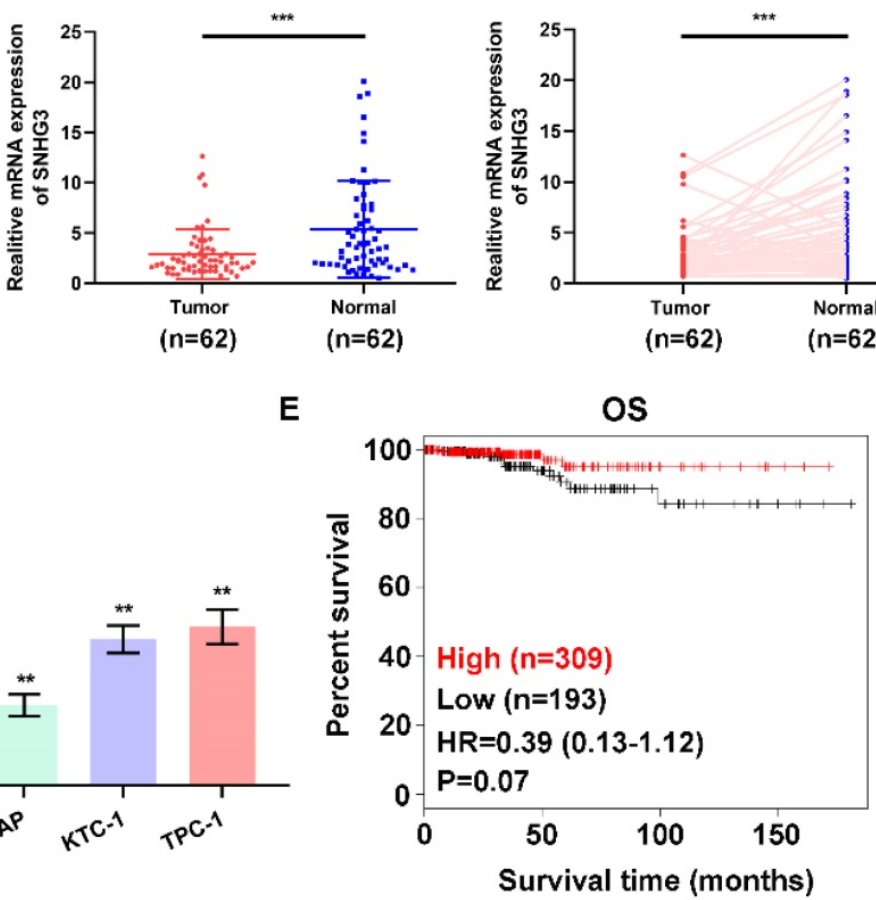

C
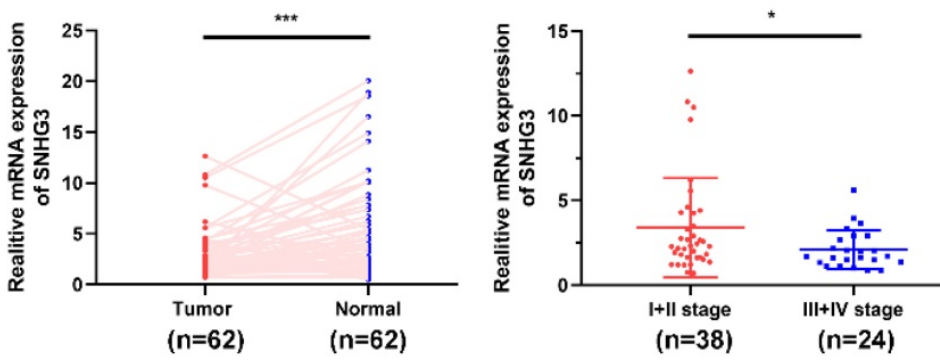

RFS

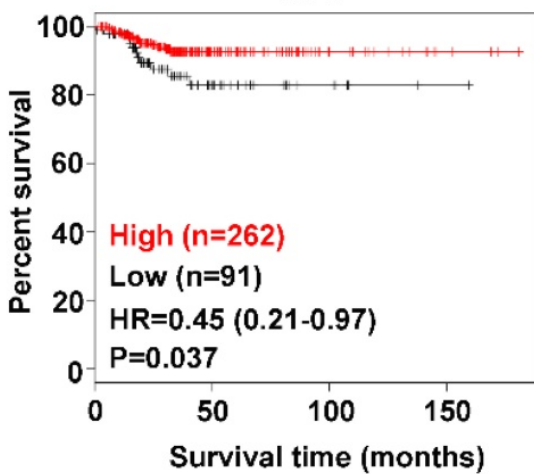

Figure 1. SNHG3 expression is generally down-regulated in PTC tissues and cells. (A) The mRNA expression of SNHG3 in PTC patients based on data from GEPIA database. (B) The RNA levels of SNHG3 was analyzed in 62 paired PTC and adjacent non-tumor tissues by quantitative RT-PCR. (C) Relative expression of SNHG3 in PTC patients with I+II tumor stage compared with patients with the advanced tumor stage. (D) SNHG3 expression in immortalized thyroid follicular cells Nthy ori 3-1 and three PTC cell lines was evaluated by RT-PCR. (E) Kaplan-Meier survival analysis for overall survival (OS) and recurrence-free survival (RFS) of PTC patients with high vs. low expression of SNHG3 in the TCGA cohort. RT-PCR results were presented as mean \pm SD. $* P<0.05$, **P $<0.01$, ***P $<0.001$. 
migrated and invaded cells in SNHG3-KO group of BCPAP and TPC-1 cells (Fig. 3A). Wound-healing assays also confirmed that knock-out of SNHG3 promoted the metastatic abilities of PTC cells in vitro (Fig. 3B). Therefore, the above findings illustrate that SNHG3 functions as a tumor suppressor during the development and progression of PTC.

\section{Silencing of SNHG3 activates the AKT/ mTOR/ERK pathway in PTC cell lines}

In order to elucidate the molecular mechanisms underlying the inhibition of SNHG3-induced malignant phenotypes, we applied RNA-seq expression profiles from TCGA THCA dataset to search for differentially expressed genes and enriched pathways between PTC patients with SNHG3-high and SHNG3 low expression. Gene set enrichment analysis (GSEA) revealed that the activation of mTOR signaling pathway and MAPK pathway were strongly related to the low expression of SNHG3 in PTC patients (Fig. 4A). We then performed western blot to detect the crucial protein kinases associated with these pathways. As displayed in Fig. 4B, inactivation of SNHG3 markedly led to the elevation of phosphorylated AKT, mTOR and ERK without affecting the total protein levels of these genes in both BCPAP and TPC-1 cells (Fig. 4B and C).
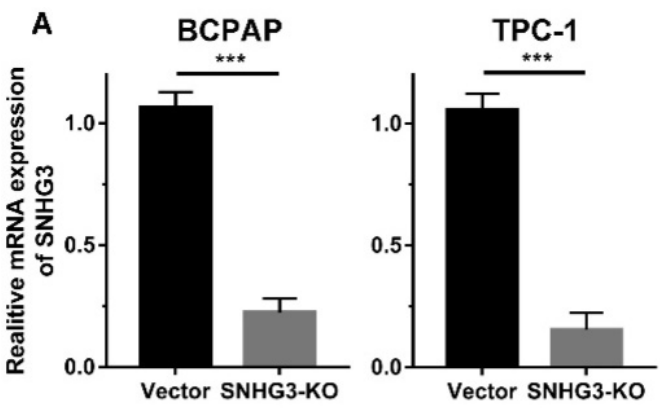

B

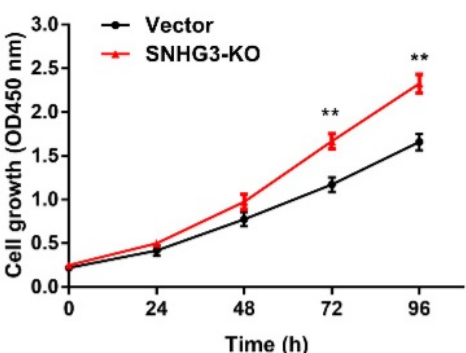

C
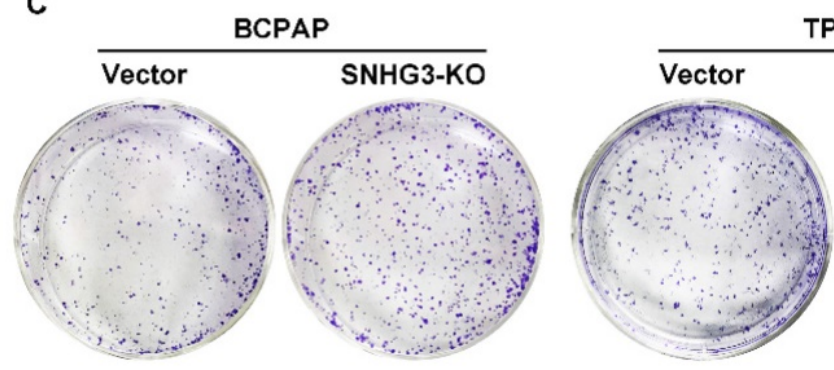

TPC-1

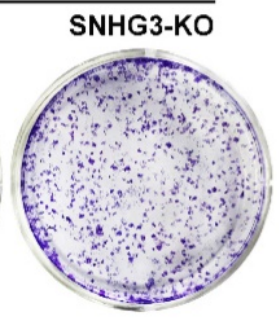

D

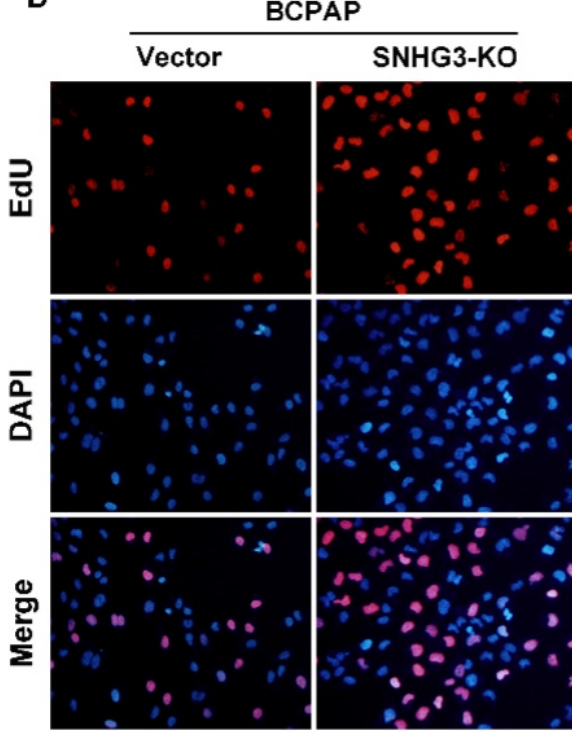

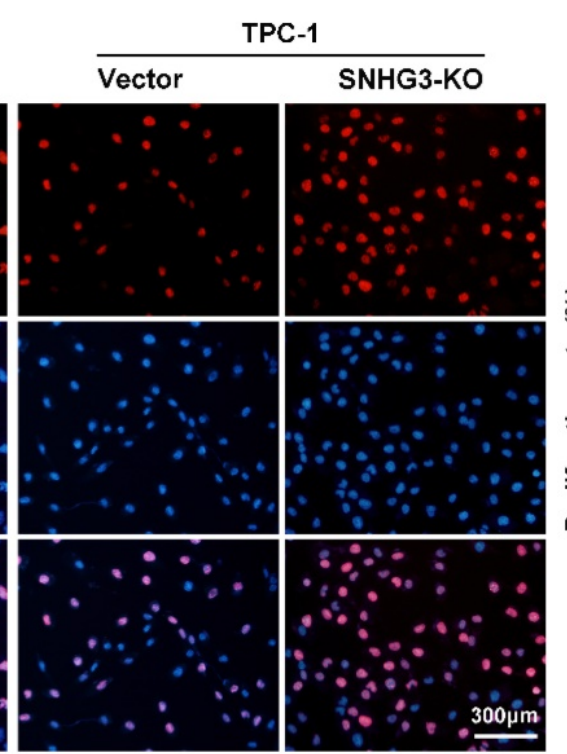

TPC-1
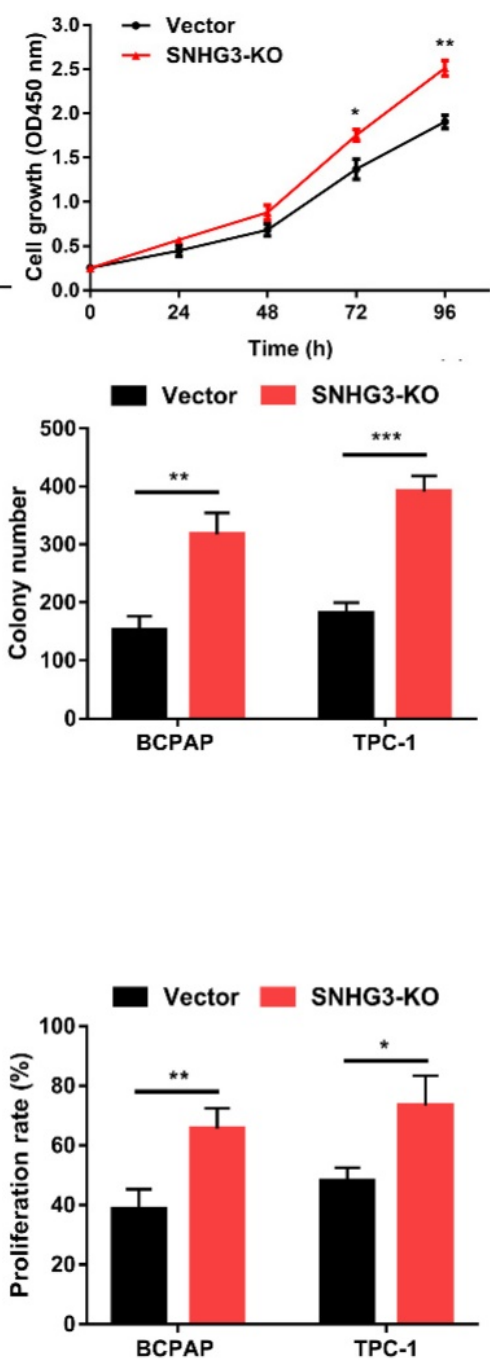

Figure 2. SNHG3 deficiency promotes PTC cells proliferation in vitro. (A) RT-PCR confirmed the knockout efficiency of SNHG3 by CRISPR/Cas 9 technology in the BCPAP and TPC-1 cells. (B) CCK-8 assays were performed to verify the functional role of SNHG3 in BCPAP and TPC-1 cell viability. (C) Representative graphs and quantification results of colonies in colony formation assay. (D) Knock-out of SNHG3 accelerated cell proliferation in PTC cell lines as determined by EdU assays. Error bars represent means $\pm S D$ from three independent experiments. $* P<0.05$, $* * P<0.01$, *** $P<0.001$. 


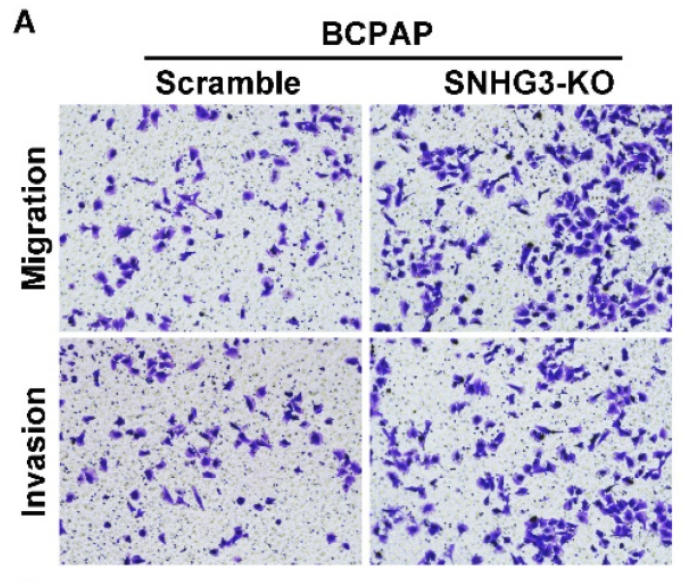

B

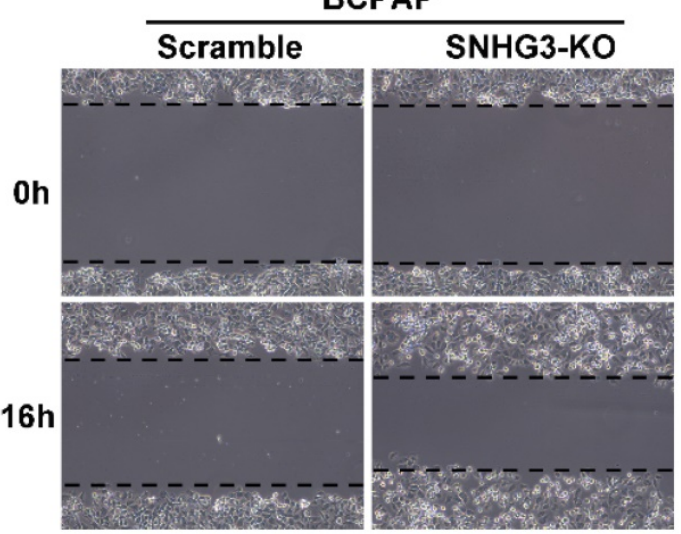

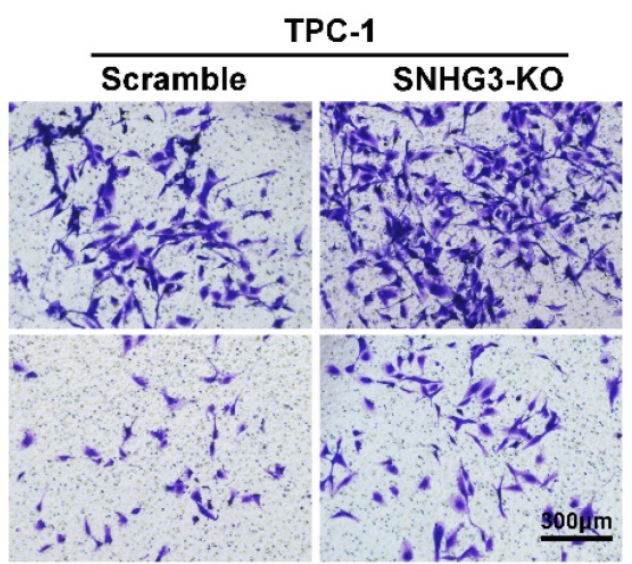

TPC-1

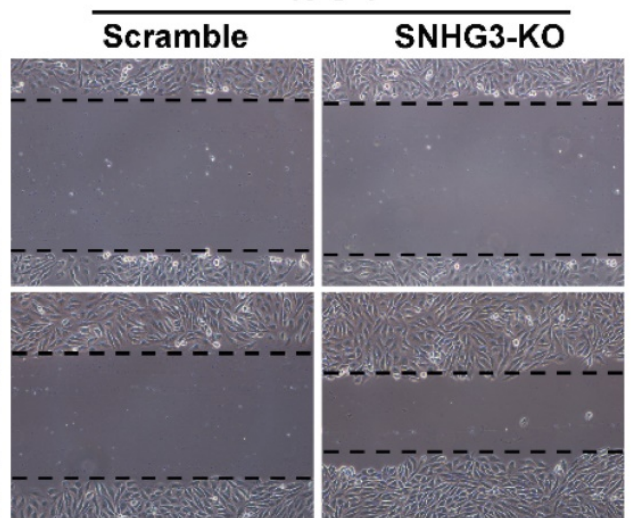

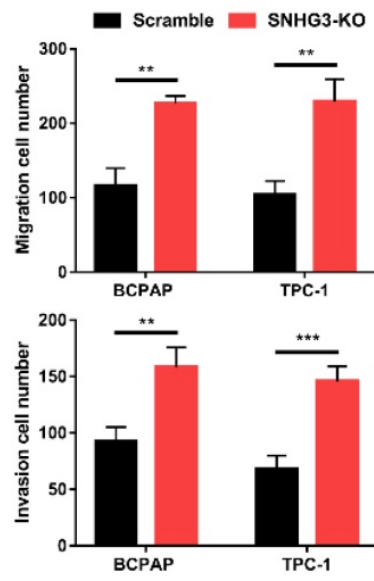

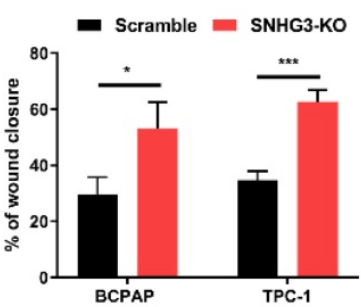

Figure 3. Inhibition of SNHG3 regulates PTC cells migration and invasion in vitro. (A) Transwell assays with or without matrigel showed that SNHG3 silencing promoted PTC cells migration (upper panel) and invasion (lower panel). (B) Wound-healing experiments confirmed the effect of SNHG3 on migration abilities in BCPAP and TPC-1 cells. Representative images (left panel) and quantification analyses (right panel) are shown. Data indicate the mean \pm SD based on three independent experiments. *P $<$ $0.05, * * \mathrm{P}<0.01, * * * \mathrm{P}<0.001$.

\section{mTOR inhibitor AZD8055 abrogates the tumor-promoting effect of SNHG3 inhibition}

To further investigate the effect of mTOR pathway in SNHG3 participated in PTC proliferation and metastasis, we introduced the mTOR inhibitor AZD8055 (Selleckchem) to conduct a series of functional rescue assays. Strikingly, treating BCPAP cells with AZD8055 prominently reversed the growth-promoting effects in PTC cells induced by the stable knock-out of SNHG3, as manifested by CCK-8 and colony-formation experiments (Fig. 5A and B). Meanwhile, transwell migration and invasion assays showed that mTOR silencing by AZD8055 was sufficient to compromise the metastatic abilities compared to SNHG3-KO cells treated with DMSO (Fig. 5C and Supplementary Fig. 3A). We carried out western blot to determine the involvement of these crucial protein during this process. As expected, the results showed that SNHG3 deficiency followed by mTOR inhibitor AZD8055 alleviated the expression of p-mTOR, p-AKT and p-ERK rather than total level of mTOR, AKT and ERK (Fig. 5D and E). Collectively, these results demonstrate that SNHG3 promotes the growth and metastasis of PTC cells partly through the activation of AKT/mTOR/ERK pathway.

\section{Knock-out of SNHG3 expedites tumor growth in vivo}

To further verify the in vitro findings, we used a xenograft model to test the tumor-suppressive role of SNHG3 in vivo. An equal amount of control and SNHG3-KO cells were subcutaneously injected into ten BALB/c nude mice. After observing for 4 weeks, we noticed that, compared with the vector group, the mean tumor volume and weight of the SNHG3-KO group were obviously higher, indicating that loss of SNHG3 facilitated the tumor growth in vivo (Fig. 6A-C). We also performed the RT-PCR in the xenografts from these two groups and the results verified the low level of SNHG3 expression in SNHG3-KO group (Fig. 6D). Additionally, immunohistochemistry staining showed that SNHG3-KO group displayed a higher positive rate of Ki-67 (Fig. 6E). 


\section{Discussion}

Researchers have focused for years to investigate the pathogenesis underlying lncRNAs mediated tumorigenesis. Emerging evidence showed that numerous lncRNAs are critical regulators of metastasis, differentiation, and metabolism of multiple human cancers $[15,16]$. Several lncRNAs have been reported to be involved in PTC tumorigenesis and progression. Yuan et al. identified a lncRNA, HOTTIP, that is upregulated in human PTC tissues and associated with growth arrest [17]. Zhu et al. described the oncogenetic role of lncRNA HOTAIR in PTC and the association between its single nucleotide polymorphisms (SNPs) and PTC risk [18]. LncRNA PTCSC3 was significantly suppressed in
PTC tumor tissue and it could affect PTC predisposition and carcinogenesis through downregulating the MMP-9 and VEGF expression via the S100A4 pathway [19]. LncRNA PVT1 regulated cell growth and cell cycle by functioning as a competing endogenous RNA (ceRNA) to regulate IGF-1 expression by sponging miR-30a [20]. Likewise, lncRNA MIAT promoted PTC cell proliferation and migration through sponging miR-212 [21]. Here, our RT-PCR results confirmed that SNHG3 was markedly silenced in PTC tissues and the expression of SNHG3 was closely related to the TNM stage of PTC patients. Besides, our study indicated that SNHG3 might serve as a novel diagnosis and prognostic biomarker for PTC patients. The above findings emphasized the important clinical value of SNHG3 in PTC patients.
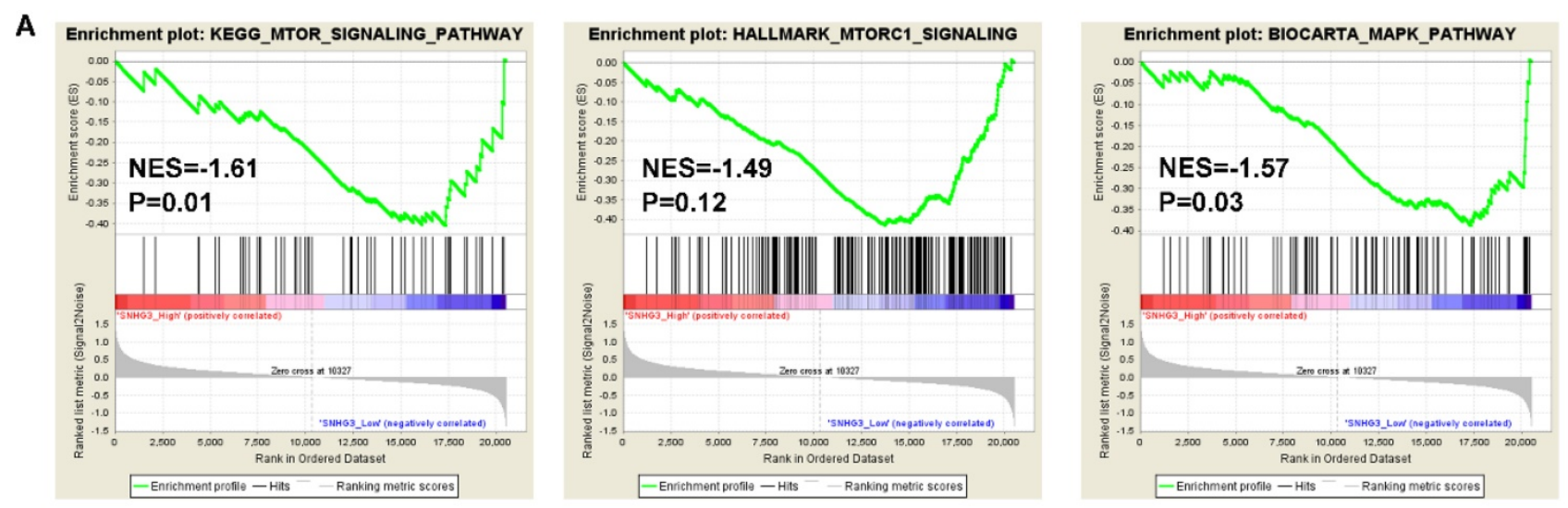

B

BCPAP

TPC-1
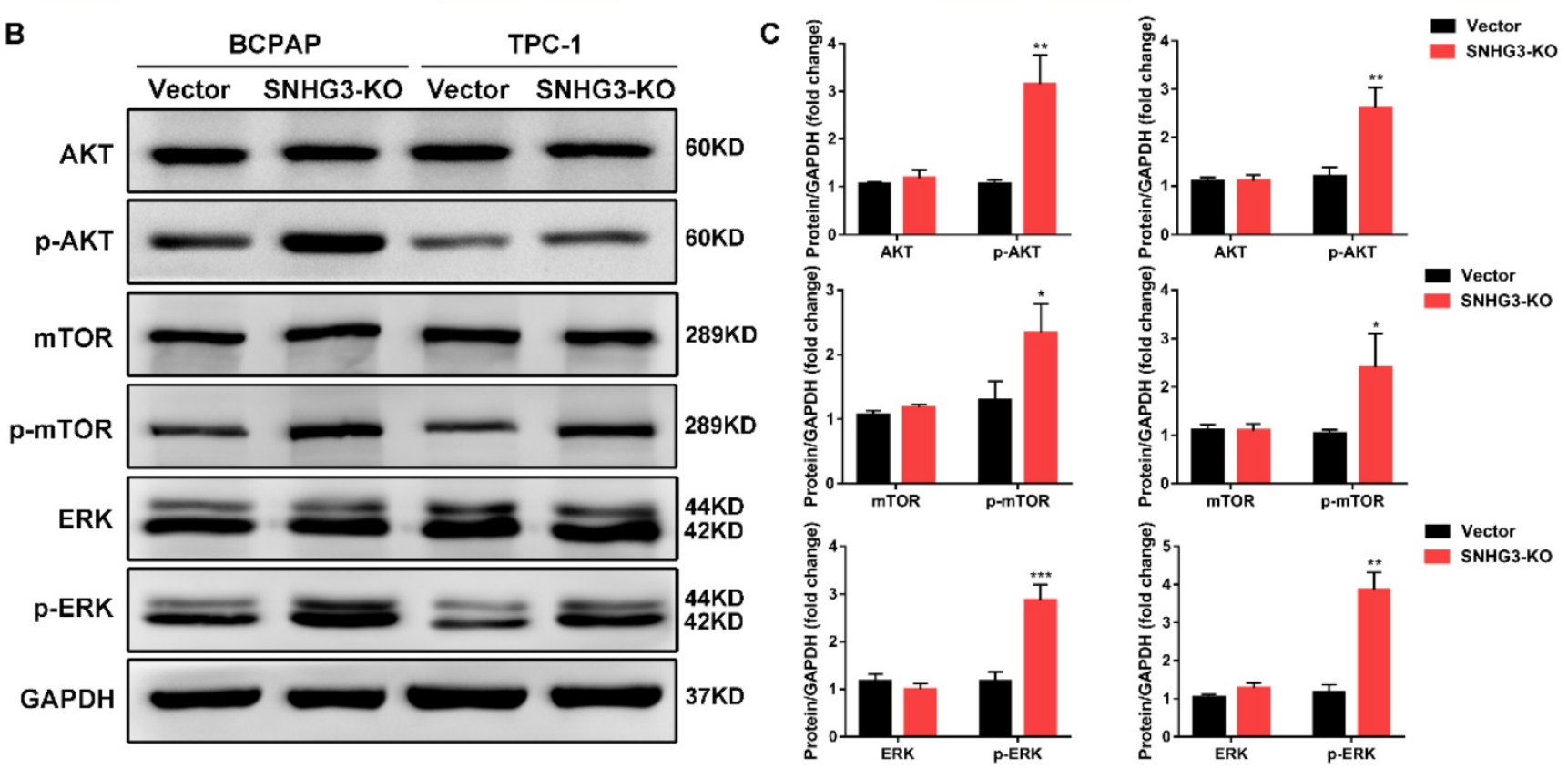

Figure 4. Bioinformatic analysis reveals that SNHG3 was involved in the AKT/mTOR/ERK pathway. (A) GSEA analysis comparing the SNHG3-high (red) and low (blue) expression subgroups of PTC patients in the TCGA dataset. Enrichment plots indicate hallmark gene sets enriched in SNHG3-low PTC patients. (B) Western blot analysis for mTOR, mTOR (Ser2481), AKT, AKT (Ser473), ERK and ERK (Thr202/Tyr204) in SNHG3 knockout and control group of BCPAP and TPC-1 cells. (C) The densitometric analysis of the western blot bands shown in Fig. 4B. The gray values are expressed as the mean $\pm \mathrm{SD}$ from three independent experiments. $* \mathrm{P}<0.05, * * \mathrm{P}<0.01, * * * \mathrm{P}<0.001$. 

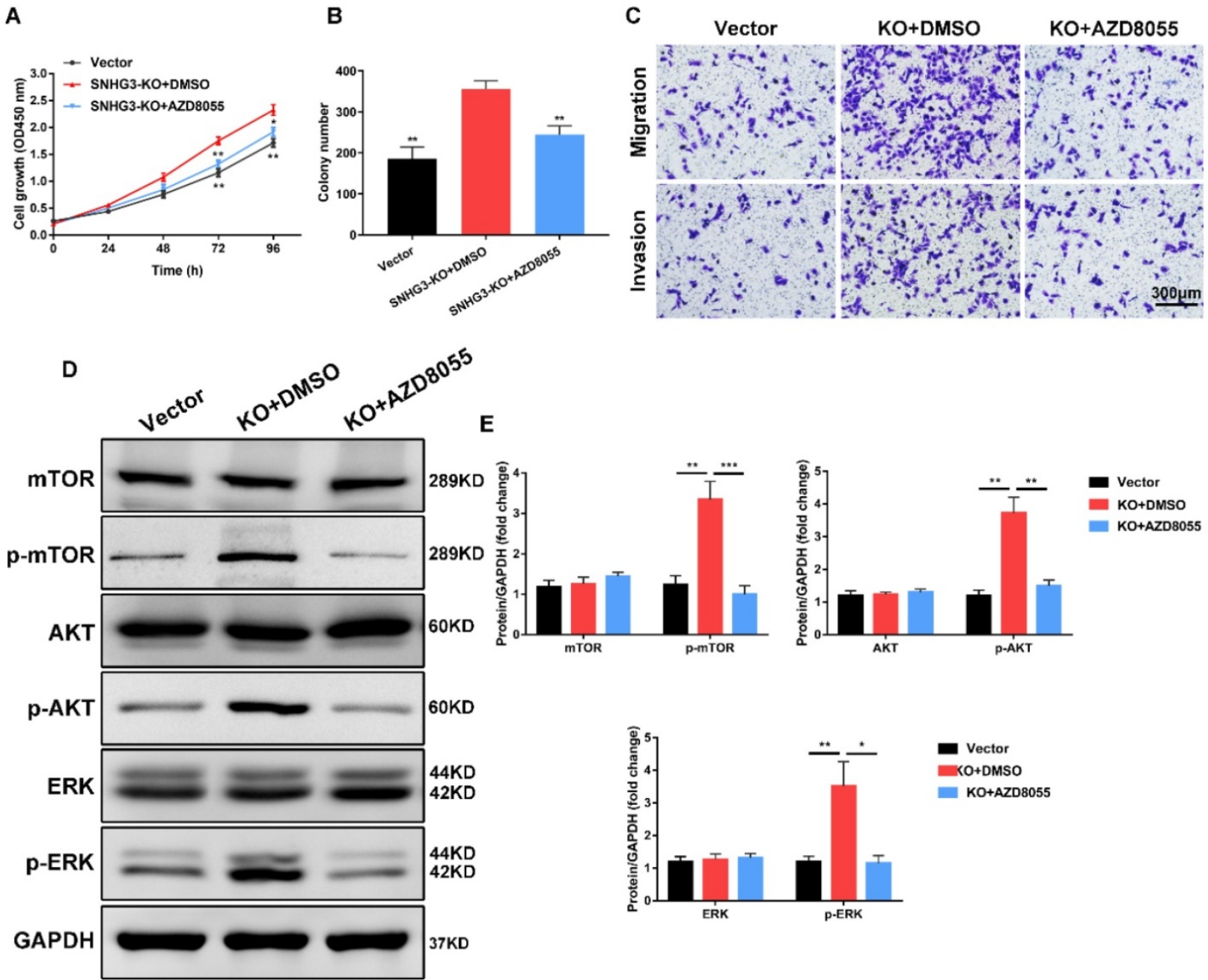

Figure 5. mTOR inhibitor AZD8055 impedes SNHG3-mediated PTC cell growth, migration and invasion. (A) BCPAP cells from control and SNHG3-KO group were treated with DMSO or $500 \mathrm{nM}$ AZD8055 for $48 \mathrm{~h}$ and the cell viability was detected by CCK-8 assays. (B) The quantification of colonies per well was counted and analyzed from PTC cells in the presence or absence of AZD8055. (C) Transwell experiments showing the effects of SNHG3 on BCPAP cell migration and invasion following treatment with AZD8055 for 48h. (D) Western blotting analysis of the protein levels of mTOR, AKT, ERK and their phosphorylation forms in BCPAP control and knockout cells with AZD8055 for 48h. (E) The densitometric analysis results of the western blot bands shown in Fig. 5D. Error bars represent three independent experiments. *P $<0.05$, $* * P<0.01$, $* * * \mathrm{P}<0.001$.

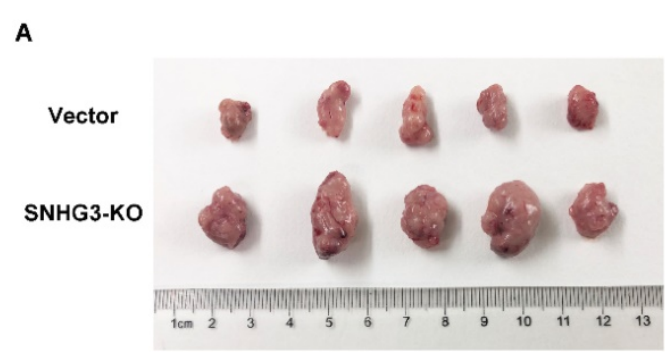

C

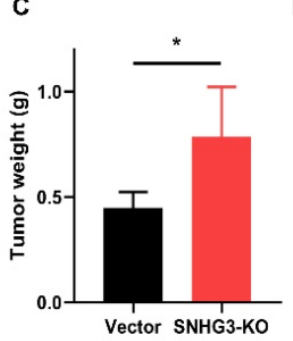

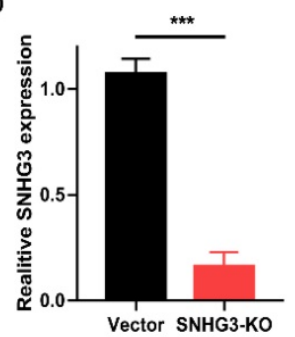

B

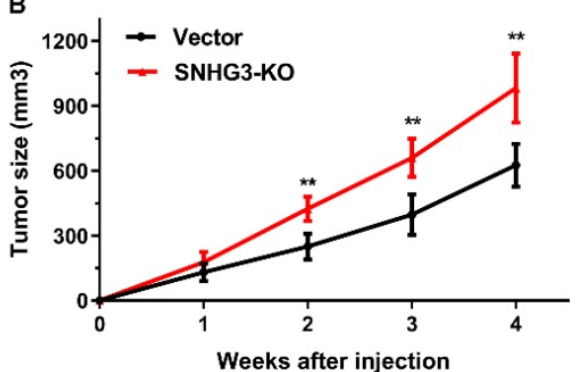

E

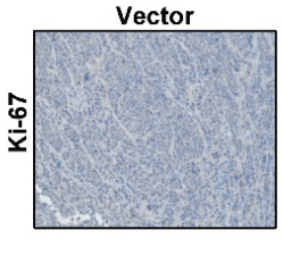

SNHG3-KO
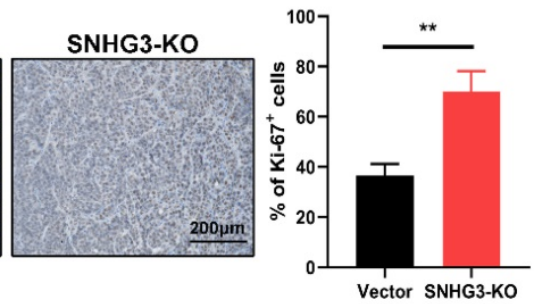

Figure 6. Knockout of SNHG3 contributes to PTC tumourigenicity in vivo. (A) Representative pictures of tumors formed in nude mouse models after injection of BCPAP cells stably transfected with vector or SNHG3-knockout. (B) Growth curve of the tumor volumes was measured every seven days in SNHG3-KO and vector group. (C) The average tumor weights were quantified from nude mice in each group. ( $n=5)$. (D) RT-PCR analysis of the expression of SNHG3 in subcutaneous tumor tissues. (E) Representative images of immunohistochemistry staining for $\mathrm{Ki}-67$ and quantification of $\mathrm{Ki}-67^{+}$cells in the indicated subcutaneous xenograft tumors. Error bars represent the mean \pm SD. $* \mathrm{P}<0.05, * * \mathrm{P}<0.01, * * * \mathrm{P}<0.001$ 
SNHG3 is a novel lncRNA potentially associated with various cancers, including lung adenocarcinoma, ovarian cancer, osteosarcoma, renal cell carcinoma, colorectal cancer and some other cancers [12,13,22-24]. SNHG3 promoted gastric cancer progression through regulating neighboring MED18 gene methylation in collaboration with EZH2 [25]. In osteosarcoma cells, overexpression of SNHG3 increased cell migration and invasion by sponging miR-151a-3p, thereby elevating the expression of its target gene RABB2A [26]. To the best of our knowledge, there were no reports regarding the expression or function of SNHG3 in PTC. Consequently, we focused on the potential involvement of SNHG3 in PTC. We further demonstrated SNHG3-deficiency by CRISPR/Cas9 significantly promoted PTC cell viability and proliferation. Besides, we validated the contributions of SNHG3 in tumor metastatic process. SNHG3silencing strengthened cell migration and invasion abilities as indicated by transwell and wound-healing assays. These phenotypes were further confirmed using in vivo xenograft mice model, in which knockout of SNHG3 contributed to PTC tumorigenesis. LncRNAs are known to participate in tumorigenesis either in a pro-tumor or anti-tumor manner $[27,28]$. However, most researches identified SNHG3 as an oncogene in various cancers. Our study, for the first time, demonstrated the anti-tumor effects of SNHG3 in PTC.

Identifying the subcellular localization of IncRNAs could provide the avenue for understanding its underlying molecular mechanism. Through cytoplasmic and nuclear fractionation assays, the results showed that SNHG3 was mainly expressed in the nucleus of PTC cells. Therefore, we postulated that SNHG3 might perform its tumorigenic role at the transcriptional, translational or post-translation level. In order to clarify the potential mechanism by which SNHG3-silencing promotes PTC cell proliferation and invasion, GSEA analysis was performed and uncovered the activation of AKT/mTOR and MAPK/ ERK signaling pathway was strongly associated with the low expression of SNHG3 in TCGA PTC patients. As expected, western blot verified that inactivation of SNHG3 distinctly led to the elevation of phosphorylated AKT, mTOR and ERK, indicating that SNHG3-silencing triggered tumor progression in PTC by modulating AKT/mTOR/ERK signaling pathway. This was confirmed by mTOR inhibitor AZD8055, which fully impedes SNHG3-mediated PTC cell growth and metastasis. AZD8055 is a first-in-class orally available ATP-competitive inhibitor of mTOR kinase activity, which selectively blocks phosphorylation of mTOR substrates, including p70S6K (S6K) and 4E-BP1 [29]. Previous report confirmed that AZD8055 exhibited an obvious tumor-inhibition effect in HRAS mutant or BRAF mutant PTC cells induced xenograft models [30]. Considered as a thyroid oncogenic pathway, $\mathrm{AKT} / \mathrm{mTOR}$ pathway is mostly hyperactivated in PTC, particularly in poorly differentiated thyroid cancer [31]. Apart from the AKT/mTOR pathway, MAPK/ERK pathway is also an important intracellular signaling pathway through a p53-dependent regulation of cell cycle [32]. MAPK signaling pathway transmits growth signals from the cell membrane to the cell nucleus and plays a crucial role in promoting PTC cell proliferation and survival [33].

In conclusion, our study demonstrated SNHG3deficiency could promote PTC cell proliferation, migration, and invasion; and contribute to PTC tumorigenesis both in vitro and in vivo through activation of AKT/mTOR/ERK pathway. Noticeably, our study for the first time uncovered the anti-tumor properties of SNHG3 in cancer and provided novel insights into the mechanisms by which SNHG3 exerts its tumor suppressor function in PTC.

\section{Supplementary Material}

Supplementary figures.

http://www.jcancer.org/v11p3492s1.pdf

\section{Acknowledgments}

This study was supported by grants from the Science and Technology Project of Henan province of China (Grant No. 192102310133) and the Medical Scientific Research Foundation of Health Department of Henan Province of China (Grant No. 201403077).

\section{Competing Interests}

The authors have declared that no competing interest exists.

\section{References}

1. Siegel RL, Miller KD, Jemal A. Cancer statistics, 2019. CA Cancer J Clin. 2019; 69: 7-34.

2. Xing M. Molecular pathogenesis and mechanisms of thyroid cancer. Nat Rev Cancer. 2013; 13: 184-99.

3. Mihailovic J, Stefanovic L, Malesevic M. Differentiated thyroid carcinoma with distant metastases: probability of survival and its predicting factors. Cancer Biother Radiopharm. 2007; 22: 250-5.

4. Batista PJ, Chang HY. Long noncoding RNAs: cellular address codes in development and disease. Cell. 2013; 152: 1298-307.

5. Huarte M. The emerging role of lncRNAs in cancer. Nat Med. 2015; 21: 1253-61.

6. Iyer MK, Niknafs YS, Malik R, Singhal U, Sahu A, Hosono Y, et al. The landscape of long noncoding RNAs in the human transcriptome. Nat Genet. 2015; 47: 199-208.

7. Zhang H, Chen Z, Wang X, Huang Z, He Z, Chen Y. Long non-coding RNA: a new player in cancer. J Hematol Oncol. 2013; 6: 37.

8. Bhan A, Soleimani M, Mandal SS. Long Noncoding RNA and Cancer: A New Paradigm. Cancer Res. 2017; 77: 3965-81.

9. Kawasaki Y, Komiya M, Matsumura K, Negishi L, Suda S, Okuno M, et al. MYU, a Target lncRNA for Wnt/c-Myc Signaling, Mediates Induction of CDK6 to Promote Cell Cycle Progression. Cell Rep. 2016; 16: 2554-64. 
10. Zhou M, Hou Y, Yang G, Zhang H, Tu G, Du YE, et al. LncRNA-Hh Strengthen Cancer Stem Cells Generation in Twist-Positive Breast Cancer via Activation of Hedgehog Signaling Pathway. Stem Cells. 2016; 34: 55-66.

11. Arisi I, D'Onofrio M, Brandi R, Felsani A, Capsoni S, Drovandi G, et al. Gene expression biomarkers in the brain of a mouse model for Alzheimer's disease: mining of microarray data by logic classification and feature selection. J Alzheimers Dis. 2011; 24: 721-38.

12. Hong L, Chen W, Wu D, Wang Y. Upregulation of SNHG3 expression associated with poor prognosis and enhances malignant progression of ovarian cancer. Cancer Biomark. 2018; 22: 367-74.

13. Huang W, Tian Y, Dong S, Cha Y, Li J, Guo X, et al. The long non-coding RNA SNHG3 functions as a competing endogenous RNA to promote malignant development of colorectal cancer. Oncol Rep. 2017; 38: 1402-10.

14. Zhang PF, Wang F, Wu J, Wu Y, Huang W, Liu D, et al. LncRNA SNHG3 induces EMT and sorafenib resistance by modulating the miR-128/CD151 pathway in hepatocellular carcinoma. J Cell Physiol. 2019; 234: 2788-94.

15. Chu J, Li H, Xing Y, Jia J, Sheng J, Yang L, et al. LncRNA MNX1-AS1 promotes progression of esophageal squamous cell carcinoma by regulating miR-34a/SIRT1 axis. Biomed Pharmacother. 2019; 116: 109029.

16. Yang L, Sun K, Chu J, Qu Y, Zhao X, Yin H, et al. Long non-coding RNA FTH1P3 regulated metastasis and invasion of esophageal squamous cell carcinoma through SP1/NF-kB pathway. Biomed Pharmacother. 2018; 106: 1570-7.

17. Yuan $\mathrm{O}$, Liu $Y$, Fan $Y$, Liu Z, Wang X, Jia M, et al. LncRNA HOTTIP promotes papillary thyroid carcinoma cell proliferation, invasion and migration by regulating miR-637. Int J Biochem Cell Biol. 2018; 98: 1-9.

18. Zhu H, Lv Z, An C, Shi M, Pan W, Zhou L, et al. Onco-lncRNA HOTAIR and its functional genetic variants in papillary thyroid carcinoma. Sci Rep. 2016; 6: 31969.

19. Jendrzejewski J, Thomas A, Liyanarachchi S, Eiterman A, Tomsic J, He H, et al. PTCSC3 Is Involved in Papillary Thyroid Carcinoma Development by Modulating S100A4 Gene Expression. J Clin Endocrinol Metab. 2015; 100: E1370-7.

20. Feng K, Liu Y, Xu LJ, Zhao LF, Jia CW, Xu MY. Long noncoding RNA PVT1 enhances the viability and invasion of papillary thyroid carcinoma cells by functioning as ceRNA of microRNA-30a through mediating expression of insulin like growth factor 1 receptor. Biomed Pharmacother. 2018; 104: 686-98.

21. Wang $\mathrm{R}$, Zhao L, Ji L, Bai L, Wen $\mathrm{Q}$. Myocardial infarction associated transcript (MIAT) promotes papillary thyroid cancer progression via sponging miR-212. Biomed Pharmacother. 2019; 118: 109298.

22. Zhang C, Qu Y, Xiao H, Xiao W, Liu J, Gao Y, et al. LncRNA SNHG3 promotes clear cell renal cell carcinoma proliferation and migration by upregulating TOP2A. Exp Cell Res. 2019; 384: 111595.

23. Liu L, Ni J, He X. Upregulation of the Long Noncoding RNA SNHG3 Promotes Lung Adenocarcinoma Proliferation. Dis Markers. 2018; 2018: 5736716.

24. Zheng S, Jiang F, Ge D, Tang J, Chen H, Yang J, et al. LncRNA SNHG3/miRNA-151a-3p/RAB22A axis regulates invasion and migration of osteosarcoma. Biomed Pharmacother. 2019; 112: 108695.

25. Xuan Y, Wang Y. Long non-coding RNA SNHG3 promotes progression of gastric cancer by regulating neighboring MED18 gene methylation. Cell Death Dis. 2019; 10: 694

26. Zheng S, Jiang F, Ge D, Tang J, Chen H, Yang J, et al. LncRNA SNHG3/miRNA-151a-3p/RAB22A axis regulates invasion and migration of osteosarcoma. Biomed Pharmacother. 2019; 112: 108695

27. Yang F, Wu Q, Zhang L, Xie W, Sun X, Zhang Y, et al. The long noncoding RNA KCNQ1DN suppresses the survival of renal cell carcinoma cells through downregulating c-Myc. J Cancer. 2019; 10: 4662-70.

28. Bo H, Fan L, Li J, Liu Z, Zhang S, Shi L, et al. High Expression of lncRNA AFAP1-AS1 Promotes the Progression of Colon Cancer and Predicts Poor Prognosis. J Cancer. 2018; 9: 4677-83.

29. Chresta CM, Davies BR, Hickson I, Harding T, Cosulich S, Critchlow SE, et al. AZD8055 is a potent, selective, and orally bioavailable ATP-competitive mammalian target of rapamycin kinase inhibitor with in vitro and in vivo antitumor activity. Cancer Res. 2010; 70: 288-98.

30. Malaguarnera R, Chen KY, Kim TY, Dominguez JM, Voza F, Ouyang B, et al. Switch in signaling control of mTORC1 activity after oncoprotein expression in thyroid cancer cell lines. J Clin Endocrinol Metab. 2014; 99: E1976-87.

31. Ringel MD, Hayre N, Saito J, Saunier B, Schuppert F, Burch H, et al. Overexpression and overactivation of Akt in thyroid carcinoma. Cancer Res. 2001; 61: 6105-11.

32. Furuya F, Lu C, Guigon CJ, Cheng SY. Nongenomic activation of phosphatidylinositol 3-kinase signaling by thyroid hormone receptors. Steroids. 2009; 74: 628-34

33. He J, Jin Y, Zhou M, Li X, Chen W, Wang Y, et al. Solute carrier family 35 member F2 is indispensable for papillary thyroid carcinoma progression through activation of transforming growth factor-beta type I receptor/apoptosis signal-regulating kinase $1 /$ mitogen-activated protein kinase signaling axis. Cancer Sci. 2018; 109: 642-55. 